\title{
Managed bumble bees are viable as pollinators in netted kiwifruit orchards
}

\author{
Brian T. Cutting ${ }^{1, *}$, Lisa J. Evans ${ }^{1}$, Ludivine I. Paugam ${ }^{2}$, Heather M. McBrydie ${ }^{3}$, Linley \\ K. Jesson ${ }^{4}$, Nelson Pomeroy ${ }^{5}$, Milena Janke ${ }^{3}$, Marine Jacob $^{2}$ and David E. Pattemore ${ }^{3}$ \\ ${ }^{1}$ The New Zealand Institute of Plant \& Food Research (PFR) Australia. c/o Queensland \\ University of Technology, GPO Box 2434, Brisbane 4001, Australia \\ ${ }^{2}$ Agrocampus Ouest, 65 Rue de Saint-Brieuc, Rennes 35000, France \\ 3 The New Zealand Institute of Plant \& Food Research, Bisley Road, Hamilton, 3214, New \\ Zealand \\ ${ }^{4}$ The New Zealand Institute of Plant \& Food Research, Crosses Rd, Parkvale, Havelock North \\ 4172, New Zealand \\ ${ }^{5}$ Nelson Pomeroy Ltd. 1010 Outram Road, Hastings 4122, New Zealand \\ *Corresponding author: brian.cutting@plantandfood.co.nz
}

\begin{abstract}
Protected cropping can increase orchard productivity; however, enclosed environments can be disruptive to pollinators. Bumble bees (Bombus terrestris) forage under covers, but industries lack guidelines for using them as pollinators. Here, conservative stocking rates for bumble bees in covered kiwifruit orchards were identified using empirical manipulation of bee density and theoretical calculations based on bee behaviour. Bumble bee pollination at two bee densities ( 24 and 72 colonies/ha) was assessed within two fully netted sections (1/25 ha each) in a Gold3 kiwifruit orchard. Predictive modelling was used to estimate total bees required for pollination while actual pollination in the two blocks was measured directly. There was no difference in flower visitation rate between stocking densities, but a $20 \%$ increase in seed set was found at high bee density. It was estimated that $90 \%$ of a Gold 3 crop could be pollinated by 914 foraging bees/ha, or between 22 and 48 colonies/ha at the strengths used in this study. Bumble bees are effective pollinators in protected cropping environments and provide a viable tool for managed pollination under nets. A theoretical approach to refining stocking rates is a good option when manipulating pollinator densities is not possible.
\end{abstract}

Keywords Protected cropping, pollination, bumble bee, kiwifruit, Gold3, crop covers, hail netting, stocking rate.

\section{INTRODUCTION}

Horticultural crops are increasingly being grown under protective covers (Castilla 2002; Cook \& Calvin 2005; Dag 2008) to optimise growing conditions and to protect the crop from pests, diseases, and extreme weather (Hanan et al. 1978; Reddy 2016). In New Zealand, approximately $20 \%$ (800 ha) of gold kiwifruit crops were covered with hail netting between 2013 and 2017, with more covers being installed each year (Beth Kyd,
Zespri Ltd., pers. comm.).

While protective covers may be beneficial for plant growth, they can inhibit the natural movement and behaviour of insects in a way that reduces their pollination efficiency. Honey bee hive strength (bee and brood numbers) can decline while deployed in covered kiwifruit even for short durations (e.g. 10 days during flowering) (unpublished research). In New Zealand, kiwifruit flowering generally co- 
occurs or immediately precedes flowering of Leptospermum scoparium (mānuka), therefore reduction of hive quality during this time may lower subsequent production of valuable mānuka honey. These concerns have been a primary driver of increases in hive rental prices for kiwifruit, and concerns about availability and sustainability of honey bees for pollination in any covered crop.

The foraging behaviour of bumble bees (Bombus terrestris) does not seem to be strongly affected by covers or enclosures; these bees are widely used for the pollination of tomatoes in glasshouses (Jones 2007; Evans 2017) and for controlled pollination for plant breeding. Bumble bees are known to visit kiwifruit flowers in covered environments and are commercially available for pollination, and thus are a promising alternative to expensive artificial pollen applications under covers. However, little information is available regarding appropriate stocking rates for bumble bees in kiwifruit orchards, much less in orchards under cover where pollinator management is of particular importance due to the exclusion of wild pollinators. Here both empirical and theoretical methods were used to develop stocking rate recommendations for bumble bees in covered Gold3 (Actinidia chinensis var. chinensis 'Zesy002') kiwifruit orchards. This is one of the principle cultivars of kiwifruit currently grown in New Zealand, and is commonly protected with hail netting.

\section{MATERIALS AND METHODS}

\section{Study site, flowering, and colony introduction}

The empirical approach involved testing two bumble bee colony stocking densities in separate, enclosed sections within a hail netting-covered Gold3 kiwifruit orchard in Te Puke, New Zealand. Internal dividers were erected to create two completely enclosed blocks of $372 \mathrm{~m}^{2}$ and $398 \mathrm{~m}^{2}$. Bumble bee colonies (established from wild-caught queens) were deployed into both blocks on 28 October 2016. The low-bee-density block was initially stocked with one colony (in $372 \mathrm{~m}^{2}$ ) for a stocking rate equivalent to 24 small colonies/ha. The high-bee-density block was stocked with three colonies; a stocking rate of equivalent to 72 colonies/ha. Deployed colonies had an average of 30 foraging bees at the time of deployment, however, forager numbers varied greatly between colonies and during the course of the experiment. Colonies were housed in wooden boxes of $0.0174 \mathrm{~m}^{3}$ internal volume with Pink $^{\otimes}$ Batts $^{\circledast}$ insulation as a nesting substrate. Boxes were fitted with trapping vestibules for calculating the number of foraging workers per colony (as per Pomeroy \& Stoklosinski 1990). Percentage flowering throughout the block was estimated by counting the number of open and closed buds on six canes in each of the two blocks. The sources of staminate kiwifruit pollen within these blocks were Actinidia chinensis var. chinensis 'M33' vines planted in several diagonal strips through the block, and pollen added to pollen dispensers (see below). Non-trial flowers in each block were hand-pollinated using liquid pollen application on 1 and 4 November 2016. Pollen applied in liquid suspension is presumed to be unavailable for subsequent movement by pollinators (Mark Goodwin, PFR, pers. comm.).

Additional colonies were added to the highbumble-bee-density block on 1 and 2 November to maintain an elevated density of foragers, and a weak colony was swapped for a stronger one on 3 November. The relative foraging strength of colonies in the high- and low-bee-density blocks was measured using trapping vestibules as follows: entrance and egress to the colony was closed for one hour during which time bees returning to the box passed through a one-way gate and were collected in a vestibule. Most foragers were trapped after one hour and these were then counted through the transparent lid in the vestibule before the colony box was reopened. The number of returning foragers captured in trapping vestibules within $1 \mathrm{~h}$ was used to measure colony strength. Under normal conditions, the majority of bees foraging from a colony would be sampled within this time. On 31 October, colony strength was measured twice during the day at $1100 \mathrm{~h}$ and $1500 \mathrm{~h}$. This frequency of sampling was thought to be disruptive to normal bumble bee behaviour, so 
subsequently foraging strength was measured only once per day on 1 November, 2 November and 4 November. Previous work has suggested that $B$. terrestris foraging activity is similar across this time period (Howlett et al. 2017a)

Because this trial was designed to present a 'best case' scenario for bumble bee pollination, each bumble bee colony was also fitted with a pollen dispenser (Koppert Biologicals). Approximately $1 \mathrm{~g}$ of fertile pollen (Pollen Plus ${ }^{\mathrm{Tm}}$ ) was added to these dispensers each day between $1000 \mathrm{~h}$ and $1500 \mathrm{~h}$.

\section{Bee activity and fruit set at different bumble bee stocking rates}

Bee activity on flowers was monitored in each block within four $3 \mathrm{~m} \times 3 \mathrm{~m}$ quadrats. Fiveminute point counts were carried out in each quadrat three times daily at approximately $0930 \mathrm{~h}, 1200 \mathrm{~h}$, and $1500 \mathrm{~h}$ from 31 October to 4 November, except on 2 November when counts were carried out at $0930 \mathrm{~h}$ and $1200 \mathrm{~h}$ only. During these periods, all bees observed interacting with flowers were recorded. Bees that were observed interacting with flowers, leaving the quadrat, and returning to focal flowers were counted only once.

For five consecutive days during flowering (31 October to 4 November), approximately 20 newly opened flowers were marked with coloured wool in one central bay in each of the high- and low-bee-density blocks. These flowers remained exposed to bumble bee visits for the duration of the flowering period, with the exception of several hours while artificial pollination was being carried out. During these periods all marked flowers were protected within paper bags to prevent unintended application of pollen.

Fruit from marked flowers were harvested and weighed on 23 March 2017 and the number of seeds was estimated using photographs of pulverised fruit and automated counting software (Nikon NIS-elements). The fruit weights and seed counts from these bumble bee pollinated flowers were compared with flowers that were hand pollinated (positive controls) using liquid pollen application (40 tagged, hand-pollinated flowers).

\section{Assessing correlation between visits and fruit set}

Each day during the experiment, small video cameras were placed directly underneath clusters of flowers to record all insect visits to newly opened flowers that were exposed to insect visitation after their removal from paper bags. Disruption to normal foraging behaviour caused by cameras is believed to be negligible as the low profile, stationary nature of cameras allows foraging bees to acclimate to their presence. After the period of video sampling, flowers were tagged and rebagged to prevent further insect visits. The period of exposure was varied to ensure that a wide range of cumulative visit numbers to individual flowers would be recorded. For some flowers, exposure to insect visits for periods greater than $24 \mathrm{~h}$ was allowed so that visitation rates on the second day after flower opening could be observed. Tagged fruit were harvested, weighed, and seeds counted.

\section{Flower handling rate and single-visit pollen deposition}

The rate at which individual bees visit flowers (flower-handling rate) was calculated by following foraging bumble bees for as long as possible on each day of the trial. The number of visits to Gold3 and 'M33' flowers was recorded for each bee followed. Single visit deposition (SVD) of pollen on stigmas of previously bagged flowers was measured. Groups of virgin flowers were un-bagged and either observed until some flowers received single visits from bumble bees or flowers were picked and presented to bees to encourage visitation. Both methods have been shown to give comparable results in onion (Allium spp.) (Howlett et al. 2017b). A subset of exposed virgin flowers did not receive bumble bee visits and were collected as controls. Collected flowers were processed by removing all stigmas and styles into microcentrifuge tubes containing $0.5 \mathrm{~mL}$ of Alexander's stain (Alexander 1980), which differentially colours staminate pollen grains and pistillate pollen grains. These samples 
were shaken for $30 \mathrm{~s}$ using a vortex to homogenise distribution of pollen in suspension, and pollen was counted in three subsamples from each tube using a haemocytometer and compound microscope.

\section{Modelling bumble bee pollination at different stocking rates}

A mathematical model of bumble bee pollination was developed, which incorporated the visitation rate of individual bees to flowers and the quantity of pollen grains deposited on kiwifruit stigmas by single bumble bee visits. Data for the model were obtained by randomly selecting sets of 15 SVD values generated in the field (data from both blocks were pooled). Each set was used to calculate the probability of a flower having 6000 pollen grains deposited after a number of visits ranging from one to 15. Previous (unpublished) work has demonstrated that Gold3 flowers require approximately 6000 pollen grains for full pollination. One thousand sets of SVD values were randomly generated and the mean value was calculated to estimate probability of full pollination at both stocking rates.

\section{RESULTS}

\section{Flowering and colony strength}

The two study blocks were established immediately adjacent to each other with the aim of minimising environmental site effects. However, differences between the two blocks were found that likely influenced flower density and plant resource allocation. The average number of buds per cane was $16.6 \pm 5.2$ standard error of the mean (SEM) canes in the high-bee-density block and $29.7 \pm 6.5$ SEM in the low-bee-density block. Also, weights of hand-pollinated control fruit were significantly higher in the high-bee-density block (high-bee-density mean $=152.5 \pm 4.3 \mathrm{~g}$ SEM, low-bee-density mean $=131.7 \pm 5.05 \mathrm{~g}$ SEM, Student's t-test $\mathrm{t}=3.12, \mathrm{df}=38, \mathrm{P}=0.003$ ). Because of these differences between the blocks, seed set, rather than fruit weight was used as a primary indicator of pollination.

Colony strength became variable when colonies were moved into the covered environment, and several colonies in the highdensity block underwent marked decline in foragers after introduction. Across all dates during the flowering period, the mean total foragers (all colonies) was $42 \pm 6.5$ in the lowdensity block and $69 \pm 4.9$ in the high-density block.

\section{Bee activity and fruit set at different bumble bee stocking rates}

No difference between the blocks was detected in terms of bumble bee activity (based on point counts), although very few bees were observed within these 5-min periods (mean high 0.93 \pm 0.20 , mean low $0.65 \pm 0.17$, Kruskal-Wallis $\mathrm{H}=0.926, \mathrm{df}=1, \mathrm{P}=0.34$ ).

Seed counts were not different for control fruit between high-bee-density and low-beedensity blocks (high-bee-density mean $=517.2 \pm$ 16.1 SEM, low-bee-density mean $=491.8 \pm 29.5$ SEM, Student's t-test $\mathrm{t}=0.75, \mathrm{df}=38, \mathrm{P}=0.46$ ) or between bee-pollinated fruit in the high-density block and the controls (control mean $=517.2 \pm$ 16.1 SEM, bumble bee pollinated mean $=490.5 \pm$ 11.3 SEM, Student's t-test $\mathrm{t}=1.14, \mathrm{df}=92, \mathrm{P}=0.26$ ), but bee-pollinated fruit contained approximately $20 \%$ more seeds in the high-density block than in the low-density block (high-bee-density mean $=490.5 \pm 11.3$ SEM, low-bee-density mean $=$ $408.5 \pm 15.3$ SEM, Student's t-test $\mathrm{t}=4.21, \mathrm{df}=156$, $\mathrm{P}<0.001)$.

\section{Assessing correlation between visits and seed \\ set}

Video recording of focal flowers resulted in $275 \mathrm{~h}$ of footage for various durations between $0700 \mathrm{~h}$ and $2000 \mathrm{~h}$ over 5 days from 31 October to 4 November 2016. Analysis of the videos showed the earliest recorded bee visit was at $0914 \mathrm{~h}$ and the latest was $1847 \mathrm{~h}$. While recorded visits spanned a period of $9.5 \mathrm{~h}$, peak visitation periods were between 1200 noon and $1600 \mathrm{~h}$. Of 47 flowers filmed for the full $0700 \mathrm{~h}$ to $2000 \mathrm{~h}$ period between 1 November and 4 November, 38 (81\%) received at least one bumble bee visit. Nineteen flowers were filmed for a two-day period and 18 (95\%) of these flowers were visited during this 
time.

In both high- and low-bee-density blocks, there was a strong relationship between the number of visits that a flower received and the number of seeds per fruit and resulting fruit weight. Because of the differences in baseline fruit weight between the blocks, only the number of seeds per fruit as a function of number of bumble bee visits is presented (Fig. 1). This relationship was not statistically different between the two blocks (ANCOVA slope $\mathrm{F} 1,155=0.59, \mathrm{P}=0.44$, intercept slope $\mathrm{F} 1,156=2.14, \mathrm{P}=0.15)$. Poisson generalised linear models were used to fit lines to these data, however the residuals suggest that these models do not explain a high proportion of the variation observed. Therefore, there are likely to be factors in addition to bee stocking rate that affected seed set in this trial.

\section{Flower visit rate}

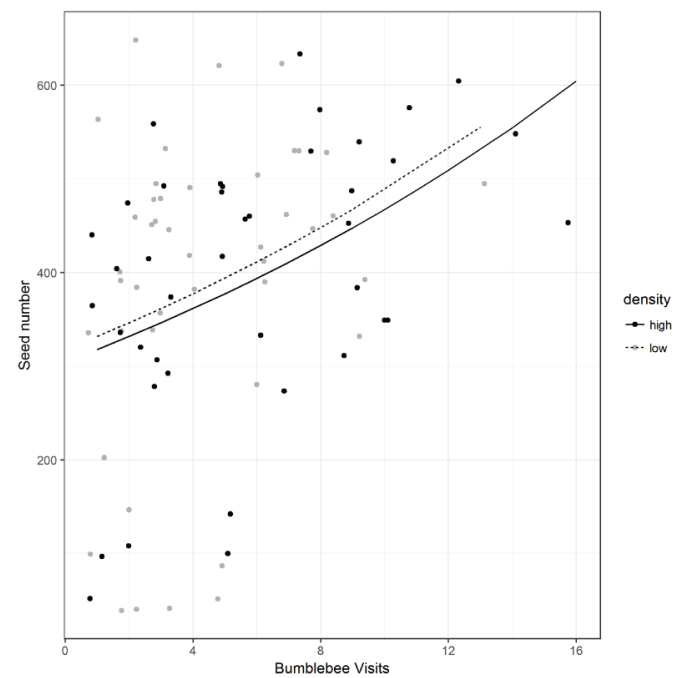

Figure 1 Number of seeds per Gold3 (Actinidia chinensis var. chinensis 'Zesy002') kiwifruit as a function of the number of bumble bee (Bombus terrestris) visits received in both high-bee-density (grey circles) and low-bee-density (black circles) blocks. Lines represent the best fit of Poisson generalised linear models for each bee density.
To assess the rate at which individual bumble bees visit flowers, 190 bees were followed. Of these, 124 were followed for at least $60 \mathrm{~s}$ and only these were used to calculate flower-handling time. Bumble bees in the low-bee-density block visited more Gold3 flowers per minute than bees in the high-density-block (mean flowers per minute high $=3.4 \pm 0.25 \mathrm{SEM}$, low $=4.7 \pm 0.29$ SEM, Kruskal-Wallis $\mathrm{H}=9.857, \mathrm{df}=1, \mathrm{P}=0.0016$ ). Of all 190 bees followed, 37 (19.5\%) visited both Gold3 and 'M33' flowers during the observation period. Single visit deposition data were collected from 45 total bumble bee visits.

\section{Single visit pollen deposition and modelling bumble bee pollination}

Single visit pollen deposition by bumble bees was not significantly different between the two blocks. Mean SVD in the high-density block was $3148 \pm$ 1408 SEM grains per visit and $943 \pm 510$ SEM in the low-density block. For all bumble bee visits (both blocks), $40 \%$ of visits $(n=18)$ deposited no pollen, 29\% $(n=13)$ deposited more than 1000 pollen grains and $11 \%(n=4)$ deposited more than 6000 pollen grains, fully pollinating flowers in a single visit. These full-pollination visits were recorded in both the high-bee-density and lowbee-density blocks.

The mathematical model developed from SVD data demonstrates the theoretical relationship between number of bumble bee visits and likelihood of full pollination of a flower (deposition of $6000+$ staminate pollen grains; Fig. 2). The model suggests that a flower has a $90 \%$ chance of being fully pollinated after 12 bumble bee visits.

\section{Calculating number of bees required.}

To estimate the number of bees required for full pollination, datasets of bumble bee flower handling rates, activity patterns and floral receptivity were combined and linked to the full pollination estimates based on SVD. It was assumed that 12 visits were required to achieve full pollination (6000 pollen grains deposited) on $90 \%$ of flowers. Bumble bee activity on flowers was observed to occur over a period of $9.5 \mathrm{~h}$; 


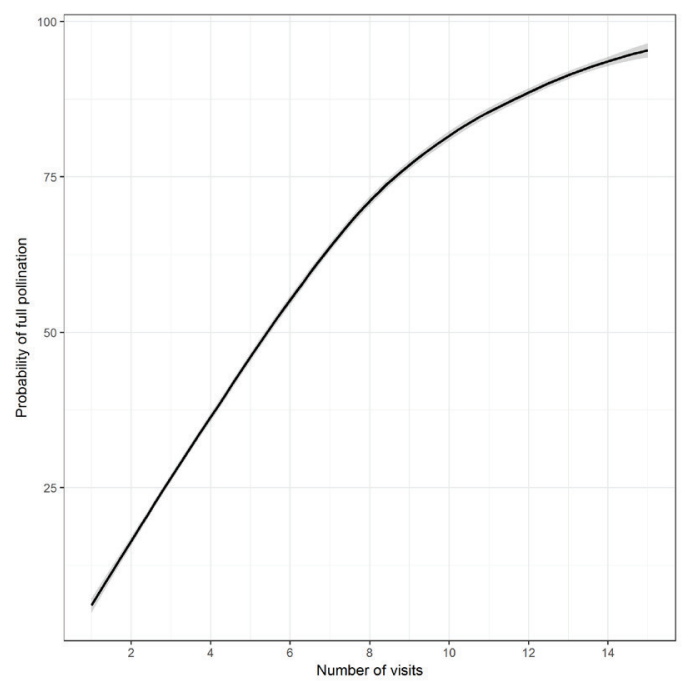

Figure 2 Probability of full pollination of Gold3 (Actinidia chinensis var. chinensis 'Zesy002') kiwifruit after $0-15$ bumble bee visits to a flower. These data were generated by sampling 15 consecutive values from field-generated data on single visit pollen deposition. Lines represent means ( \pm 95\% CI in grey) after 1000 iterations.

however, peak activity was about $4 \mathrm{~h}$ in duration. To conservatively estimate the number of bees required, $4 \mathrm{~h}$ of daily activity was assumed. Flowers are normally receptive for a period of at least 2 days and, therefore, are exposed to at least $8 \mathrm{~h}$ of peak bumble bee activity. Bees followed for $60 \mathrm{~s}$ or more visited on average 4.09 flowers per minute, so it was estimated that a foraging bumble bee can visit 245 female flowers per hour.

At this rate, to achieve 12 visits to all of the flowers in the low-bee-density block over 2 days of 'peak bumble bee activity', it was estimated that 73 bees would be required for full pollination. On any given day during flowering, no more than $50 \%$ of flowers were observed to open over any 2-day period, meaning that an estimated 37 bees would still be theoretically adequate to achieve the number of visits required. This equates to 914 foraging bumble bees per ha assuming 30 flowers per $\mathrm{m}^{2}$.

\section{DISCUSSION}

The model developed here based on SVD data estimated that $90 \%$ of flowers were pollinated after 12 bumble bee visits. Furthermore, 11\% of visits deposited more than 6000 pollen grains in a single visit. These values along with the assessment of colony strengths, and average flower densities of 30 per $\mathrm{m}^{2}$ were used to conservatively estimate that 914 foraging bumble bee workers would be sufficient to fully pollinate a covered, 1-ha kiwifruit block. This density is higher than the stocking rate estimated for open 'Hayward' kiwifruit orchards (Pomeroy \& Fisher 2002), but represents a conservative approach for a different cultivar. Using colonies of similar strength to those in this study, this would require $22-48$ colonies per ha. This estimate is supported by the comparison of fruit set and quality between the two blocks, one with a high bee density equivalent to 72 colonies per ha and the other a low-density equivalent to 24 colonies hives per ha. At the high stocking rate, bumble bee pollinated Gold3 kiwifruit had the same mass and seed count as hand pollinated controls, indicating that the bumble bees were achieving maximum pollination. At the low stocking rate, bumble bee pollinated fruit had 20\% fewer seeds than controls, suggesting that the actual stocking rate required in this case was between the two rates tested.

The authors consider that the stocking rates calculated in this study represent conservative estimates, and stocking rates may be refined downward in further experiments. Manipulation of colonies during this trial (with trapping vestibules) is likely to have slightly decreased the visitation rate of bees to flowers. Furthermore, the calculations were limited to periods of peak foraging activity; however foraging occurs at a lower level over a much longer time frame. Lastly, the model developed here essentially assumes that any flower receiving fewer than 6000 pollen grains is not adequately pollinated, when in reality, many of these flowers would develop into adequately sized fruit.

Importantly, stocking rate results from the calculated theoretical approach (using visitation rate, SVD, activity and receptivity patterns) 
applied here are aligned with results from the empirical approach of directly manipulating stocking rates. In most crops, honey bee and bumble bee stocking rates are notoriously difficult to calculate because of the challenge of finding single-pollinator sites, and the risk associated with pollination failure if experimental stocking rates are too low. Theoretical approaches may present a useful option for estimating appropriate stocking rates without directly manipulating pollinator abundance.

\section{ACKNOWLEDGEMENTS}

The authors would like to thank Colin Limmer for orchard access and field support, Beth Kyd and Mary Black (Zespri Ltd.) for assistance with experimental setup, and Brad Howlett and Warrick Nelson (PFR) for thoughtful advice on this manuscript. The manuscript further benefitted from the thoughtful comments of two reviewers. The project was funded through the Ministry of Business, Innovation and Employment programme number C11X1309 ('Bee minus to Bee plus and Beyond: Higher yields from smarter growth-focused pollination systems') and co-funding support by Zespri Group Limited, Summerfruit New Zealand (SNZ), Avocados NZ and the Foundation for Arable Research.

\section{REFERENCES}

Alexander, MP 1980. A versatile stain for pollen, fungi, yeast, and bacteria. Stain Technology 55: 13-18.

Castilla N 2002. Current situation and future prospects of protected crops in the Mediterranean region. Proceedings of the International Symposium on Mediterranean Horticulture: Issues and Prospects, pp. 135147.

Cook R, Calvin L 2005. Greenhouse tomatoes change the dynamics of the North American fresh tomato industry. Economic Research Report No. ERR-2. United States Department of Agriculture. 86 p. https://www.ers.usda. gov/publications/pub-details/?pubid $=45477$

Dag A 2008. Bee pollination of crop plants under environmental conditions unique to enclosures. Journal of Apicultural Research 47: 162-165.

Evans E 2017. From humble bee to greenhouse pollination workhorse: can we mitigate risks for bumble bees? Bee World, 94: 34-41.

Hanan JJ, Holly WD, Goldsberry KL 1978. Greenhouse management. Springer Berlin.

Howlett BG, Read SFJ, Jesson LK, Benoist A, Evans LE, Pattemore DE 2017a. Diurnal insect visitation patterns to 'Hayward' kiwifruit flowers in New Zealand. New Zealand Plant Protection 70: 52-57.

Howlett BG, Evans LJ, Pattemore DE, Nelson WR 2017b. Stigmatic pollen delivery by flies and bees: Methods comparing multiple species within a pollinator community. Basic and Applied Ecology 19: 19-25.

Jones JB 2007. Tomato plant culture: In the field, greenhouse, and home garden. CRC Press, Boca Raton, Florida, 216 p.

Pomeroy N, Fisher RM 2012. Pollination of kiwifruit (Actinidia deliciosa) by bumble bees (Bombus terrestris): effects of bee density and patterns of flower visitation., New Zealand Entomologist 25(1): 41-49

Pomeroy N, Stoklosinski SR 1990. Measuring the foraging strength of bumble bee colonies. In: Shriva P ed. Proceedings of the XIth Congress of the International Union for the Study of Social Insects: 252-253. Bangalore, India.

Reddy PP 2016. Sustainable crop protection under protected cultivation. Springer, Singapore, $434 \mathrm{p}$. 